\title{
Volumetric invariants and operators on random families of Banach spaces
}

\author{
by \\ Piotr Mankiewicz (Warszawa) and \\ Nicole TomcZaK-Jaegermann (Edmonton) \\ Dedicated to Olek Petczyniski on the occasion of his 70th birthday \\ with lots of thanks for being a mentor \\ and such an exciting friend all these years
}

\begin{abstract}
The geometry of random projections of centrally symmetric convex bodies in $\mathbb{R}^{N}$ is studied. It is shown that if for such a body $K$ the Euclidean ball $B_{2}^{N}$ is the ellipsoid of minimal volume containing it and a random $n$-dimensional projection $B=P_{H}(K)$ is "far" from $P_{H}\left(B_{2}^{N}\right)$ then the (random) body $B$ is as "rigid" as its "distance" to $P_{H}\left(B_{2}^{N}\right)$ permits. The result holds for the full range of dimensions $1 \leq n \leq \lambda N$, for arbitrary $\lambda \in(0,1)$.
\end{abstract}

0. Introduction. The systematic study of random quotients of $l_{1}^{n}$ 's begun with the acclaimed solution by E. D. Gluskin of the problem of diameters of Minkowski compacta [G1]. Soon after, several papers followed in which optimal estimates of basic constants or symmetry constants for such quotients were given [S1], [G2], [M1]. It was J. Bourgain who first used this line of argument in the context of general finite-dimensional Banach spaces, and this approach was further developed by the authors in a series of papers (cf. e.g. [MT1], [MT2]). We refer the reader to the survey [MT3] for more information on the subject.

The present paper is a further development of the study, begun in [MT4], of geometric and linear properties of families of "random" projections of symmetric convex bodies. Here we investigate symmetry properties of such random projections in terms of the existence of well bounded operators on associated Banach spaces, and we relate these properties to average geometric distance of the projections to the Euclidean ball.

2000 Mathematics Subject Classification: Primary 46B20.

P. Mankiewicz was partially supported by KBN grant no. 2 P03A 01319.

N. Tomczak-Jaegermann holds the Canada Research Chair in Geometric Analysis. 
We consider the concept of "randomness" in the following context, natural in the asymptotic theory of finite-dimensional normed spaces. Given a symmetric convex body $K$ embedded in $\mathbb{R}^{N}$ in a certain natural way, for any $1 \leq n \leq N$ the randomness on the set of all rank $n$ orthogonal projections in $\mathbb{R}^{N}$ is determined by the Grassmann manifold $G_{N, n}$ of all $n$-dimensional subspaces of $\mathbb{R}^{N}$, with the normalized Haar measure $\mu_{N, n}$.

Our main result has a dichotomous character similar to the results in [MT4]. Intuitively speaking it shows that for an arbitrary symmetric convex body $K \subset \mathbb{R}^{N}$ as above, if a random orthogonal projection $B=P(K)$ of $K$ is "far" from the Euclidean ball, then the body $B$ is as "rigid" as it can be and every well bounded operator on $B$ must be "close" to a multiple of the identity. In particular every projection on $B$ of rank proportional to $\operatorname{dim} B$ must have the norm as large as it can be (with the given distance to the Euclidean ball), and the basis constant and other symmetry invariants of $B$ must be of the same order.

Let us now describe the content of the paper in more detail. Section 1 contains all basic definitions as well as the statements of the results. Here let us only mention that the rigidity of the body in the above sense is measured by the so-called mixing constant, which is known to control how "close" well bounded operators are to a multiple of the identity. In more precise terms, our main theorem (Theorem 1.1) provides a lower estimate for the mixing constant of random orthogonal projections of $K$ by the average geometric distance of the projections of $K$ to the corresponding projections of the Euclidean ball. Thus, this and other results have similar form to those in [MT4], and, in particular, they are valid in the full range of dimensions $1 \leq n \leq \lambda N$, for any $0<\lambda<1$, which was achieved in [MT4] for the first time in this type of problems.

Our result, like many earlier results for random quotients of general normed spaces, makes an essential use (in Theorems 1.3 and 1.4) of a certain volumetric invariant of convex bodies, which was also used in other papers (cf. [MT3] and the references therein, and [MT4]). In fact, in the earlier papers the whole series of similar invariants was introduced, depending on a particular problem on hand. A new element of the present paper, which might be of an independent interest, is a realization that most of the invariants of this type are in fact equivalent. This is closely connected with the deep fact of existence of $M$-ellipsoids, and is described in Section 2.

Section 3 describes a reformulation of Theorem 1.3 in the Gaussian setting, and Sections 4 and 5 are devoted to the details of the proof of the corresponding Gaussian problem (Theorem 3.4). The general strategy of this proof goes back to papers [S1], [S2] and [M2], which treated the case of the unit ball in $l_{1}^{n}$, and to [B] and [MT2], for the general case. All these results were based on a probabilistic approach, but the underlying proba- 
bilities were not rotation invariant. We also refer the reader to the survey [MT3] for details and further references.

1. Mixing operators on families of random projections. We shall use the standard notation in the asymptotic theory of Banach spaces as for example in $[\mathrm{Pi}],[\mathrm{T}]$ and $[\mathrm{MiS} 1]$. In particular, the $n$-dimensional real Euclidean space is denoted by $\left(\mathbb{R}^{n},\|\cdot\|_{2}\right)$. For $1 \leq k \leq n, G_{n, k}$ denotes the Grassmann manifold of $k$-dimensional linear subspaces of $\mathbb{R}^{n}$ equipped with the normalized rotation invariant measure $\mu_{n, k}$. By a symmetric convex body in $\mathbb{R}^{n}$ we always mean a centrally symmetric convex body. For two such bodies $B_{1}, B_{2}$ the geometric distance is defined by

$$
d_{\mathrm{g}}\left(B_{1}, B_{2}\right)=\min \left\{\lambda_{2} / \lambda_{1} \mid \lambda_{1} B_{1} \subset B_{2} \subset \lambda_{2} B_{1}\right\} .
$$

We denote by $\lceil\varrho\rceil$ for $\varrho \in \mathbb{R}$ the smallest integer greater than or equal to $\varrho$. Let us recall the definitions of a mixing operator and mixing constant, the first of which is due to S. J. Szarek [S2] (however, this notion was implicit in [G2], [S1] and almost explicit in [M1]), while the second notion was introduced in [MT3]. For $0<\kappa \leq 1 / 2$ and $\alpha>0$ we say that a linear operator $T: \mathbb{R}^{n} \rightarrow \mathbb{R}^{n}$ is $(\kappa n, \alpha)$-mixing provided that there exists a linear subspace $E \subset \mathbb{R}^{n}$ with $\operatorname{dim} E=\lceil\kappa n\rceil$ satisfying

$$
\operatorname{dist}(T x, E)=\left\|P_{E^{\perp}} T x\right\|_{2} \geq \alpha\|x\|_{2}
$$

for every $x \in E$. The set of all $(\kappa n, \alpha)$-mixing operators on $\mathbb{R}^{n}$ is denoted by $\operatorname{Mix}_{n}(\kappa n, \alpha)$. For a symmetric convex body $B \subset \mathbb{R}^{n}$ the mixing constant of $B$ is defined by

$$
\mathbf{m}(B, \kappa)=\inf \left\{\|T: B \rightarrow B\| \mid T \in \operatorname{Mix}_{n}(\kappa n, 1)\right\}
$$

for $\lceil\kappa n\rceil \leq n / 2$ and $\mathbf{m}(B, \kappa)=\infty$ otherwise.

It follows directly from the definition that for every $0<\kappa \leq 1 / 2$,

$$
d_{\mathrm{g}}\left(B, B_{2}^{n}\right) \geq \mathbf{m}(B, \kappa) \geq d_{\mathrm{g}}\left(B, B_{2}^{n}\right)^{-1}
$$

and

$$
\mathbf{m}(B, \kappa)=\mathbf{m}\left(B^{\circ}, \kappa\right)
$$

where $B^{\circ}$ denotes the polar of $B$.

It is known that the invariant $\mathbf{m}(B, \kappa)$ is a good measure of the rigidity of the body $B$. Namely, for a well bounded operator acting on $B$, it controls how "close" this operator is to a multiple of the identity (cf. [MT3, Theorem 16]).

For a symmetric convex body $B \subset \mathbb{R}^{n}$ we shall consider (as in [MT4]) the expected Euclidean distance, defined for $1 \leq m \leq n$ by

$$
\mathbb{E} d_{\mathrm{g}}(B, m)=\int_{G_{n, m}} d_{\mathrm{g}}\left(P_{L}(B), P_{L}\left(B_{2}^{n}\right)\right) d \mu_{n, m}(L) .
$$


Clearly, $\mathbb{E} d_{\mathrm{g}}(B, m)$ is monotone in $m$, that is,

$$
\mathbb{E} d_{\mathrm{g}}\left(B, m_{1}\right) \geq \mathbb{E} d_{\mathrm{g}}\left(B, m_{2}\right) \quad \text { whenever } m_{1} \geq m_{2} \geq 1 .
$$

Now let us recall important concepts connected to Dvoretzky's theorem ([Mi2], cf. also [MT4, (1.1)-(1.3)], and for the fundamentals of the theory also [MiS1]). Let $K \subset \mathbb{R}^{N}$ be a symmetric convex body. We define a transition dimension $k^{*}(K)$ (cf. [MT4, (1.1)-(1.3)]) as the largest dimension $k$ such that the set

$$
\mathcal{A}_{k}=\left\{H \in G_{N, k} \mid(1 / 2) M_{K}^{*} P_{H}\left(B_{2}^{N}\right) \subset P_{H}(K) \subset 2 M_{K}^{*} P_{H}\left(B_{2}^{N}\right)\right\}
$$

has measure

$$
\mu_{N, k}\left(\mathcal{A}_{k}\right) \geq 1-e^{-k}
$$

where $M_{K}^{*}$ is defined by

$$
M_{K}^{*}=\int_{S^{N-1}}\|x\|_{K^{\circ}} d \mu(x)
$$

We have

$$
c^{\prime}\left(M_{K}^{*} / a\right)^{2} N \leq k^{*}(K) \leq C^{\prime}\left(M_{K}^{*} / a\right)^{2} N,
$$

where $a>0$ denotes the smallest number such that $K \subset a B_{2}^{N}$, and $C^{\prime} \geq$ $c^{\prime}>0$ are numerical constants.

To describe in a uniform way some concentration of measure phenomena which, for a fixed symmetric convex body $K \subset \mathbb{R}^{N}$, depend on dimension $1 \leq n \leq N$, we define the concentration function

$$
\alpha_{K}(n)= \begin{cases}\exp (-n) & \text { for } k^{*}(K) \leq n \leq N, \\ \exp \left(-k^{*}(K)\right) & \text { for } 1 \leq n \leq k^{*}(K) .\end{cases}
$$

We are now ready to state the main result of this paper.

Theorem 1.1. For every $0<\lambda<1,0<\kappa \leq 1 / 2$ and $0<\eta<\kappa / 2$ there exists $c=c(\lambda, \kappa, \eta)>0$ with the following property: Let $K \subset \mathbb{R}^{N}$ be a symmetric convex body such that the Euclidean unit ball $B_{2}^{N}$ is the ellipsoid of minimal volume containing $K$. Let $\eta^{-1} \leq n \leq \lambda N$ and set $m=\lceil(\kappa-\eta) n\rceil$. Then the set of all $H \in G_{N, n}$ satisfying

$$
\mathbf{m}\left(P_{H}(K), \kappa\right) \geq c \mathbb{E} d_{\mathrm{g}}\left(P_{H}(K), m\right)
$$

has measure larger than or equal to $1-2 \alpha_{K}(n)$. In particular

$$
\int_{G_{N, n}} \mathbf{m}\left(P_{H}(K), \kappa\right) d \mu_{N, n}(H) \geq c \mathbb{E} d_{\mathrm{g}}(K, m) .
$$

REMARK. Note that in view of (1.4), by a standard duality argument, the analogous theorem holds for every symmetric convex body $B$ in $\mathbb{R}^{N}$ for which $B_{2}^{N}$ is the ellipsoid of maximal volume inscribed in $B$, with projections 
$P_{H}(K)$ replaced by sections $H \cap B$ and $\mathbb{E} d_{\mathrm{g}}\left(P_{H}(K), m\right)$ replaced by

$$
\mathbb{E} d_{\mathrm{g}}^{\circ}(B, m)=\int_{G_{n, m}} d_{\mathrm{g}}\left(L \cap B, L \cap B_{2}^{n}\right) d \mu_{n, m}(L) .
$$

REMARK. Recall that by John's theorem every symmetric convex body $B \subset \mathbb{R}^{N}$ admits a position $u(B)$ (where $u$ is a linear automorphism of $\mathbb{R}^{N}$ ) such that $B_{2}^{N}$ is the unique ellipsoid of minimal volume containing $u(B)$. Thus, in the theorem above, the assumption on the ellipsoid of minimal volume may be viewed as the choice of a proper position $K=u(B)$ in the case of an arbitrary symmetric convex body $B$. It provides the norm with respect to which the mixing constants are computed and induces the Haar measure on the family of projections of the body $K$.

Let us note that in Theorem 1.1 the assumption that $B_{2}^{N}$ is the ellipsoid of minimal volume containing $K$ and the drop of dimension to $m$ in the conclusion are both essential. This can be shown by the same examples as in [MT4, Examples 2.4 and 2.5 respectively]. We sketch the argument only for the latter case, and refer the reader to [MT4] for details.

ExAmple 1.2. Let $l \geq 1$. Set $N=4 l, n=2 l$ and $\kappa=1 / 2$. Let $\mathbb{R}^{N}=$ $\mathbb{R}^{3 l} \oplus \mathbb{R}^{l}$ and set

$$
K=\operatorname{conv}\left\{B_{1}^{3 l} \oplus B_{2}^{l}\right\} .
$$

For most $H \in G_{N, n}$ the projections $P_{H}(K)$ are isomorphic, up to a numerical constant $C>0$, to the orthogonal sum $Z=X_{H} \oplus B_{2}^{l}$, where $X_{H}$ is a suitable $l$-dimensional projection of $B_{1}^{3 l}$. Let $P_{1}: Z \rightarrow Z$ be the orthogonal projection onto $X_{H}$ and $J: X_{H} \rightarrow B_{2}^{l}$ be the formal identity map. It follows directly from the definition that $J P_{1}: Z \rightarrow Z$ is in $\operatorname{Mix}_{n}(1 / 2,1)$. Since $X_{H} \subset B_{2}^{N}$ the norm of $J P_{1}: Z \rightarrow Z$ is bounded by 1 . This implies $\mathbf{m}\left(P_{H}(K), 1 / 2\right) \leq C$.

On the other hand, if $m=\lceil(1+\eta) l\rceil$ for $\eta>0$ then $\mathbb{E} d_{\mathrm{g}}\left(P_{H}(K), m\right) \geq$ $c \sqrt{n}$ for some $c=c(\eta)>0$.

In a complete analogy to the approach in [MT4], inequality (1.11) for the mixing invariant of a typical projection of a body $K$ will be obtained in two independent steps. The first will provide a lower estimate for the invariant in terms of a certain volumetric invariant of the projections of the body, while the second will relate the volumetric invariant to the Euclidean distances of random projections. These steps are the content of Theorems 1.3 and 1.4.

For a Borel subset $B$ of a $k$-dimensional subspace $E \subset \mathbb{R}^{n}$ we denote by $|B|$ the $k$-dimensional Lebesgue measure of $B$. Following [MT4] we consider a volumetric invariant which plays an essential role in our considerations. 
For a symmetric convex body $B \subset \mathbb{R}^{n}$ and $1 \leq l \leq k \leq n$, let

$$
W(B, k, l)=\inf _{E} \sup _{F}\left(\frac{\left|B_{2}^{l}\right|}{|B \cap F|}\right)^{1 / l},
$$

where the infimum is taken over all $k$-dimensional subspaces $E \subset \mathbb{R}^{n}$ and the supremum is taken over all $l$-dimensional subspaces $F \subset E$.

To state the next inequalities in a compact form we need one more notation. For a symmetric convex body $K \subset \mathbb{R}^{N}$ and $1 \leq n \leq N$, let

$$
\varphi_{K}(n)= \begin{cases}(n / N)^{1 / 2} & \text { for } k^{*}(K) \leq n \leq N, \\ \left(k^{*}(K) / N\right)^{1 / 2} & \text { for } 1 \leq n \leq k^{*}(K) .\end{cases}
$$

Theorem 1.1 is a direct consequence of the following two results.

Theorem 1.3. For every $0<\lambda<1,0<\kappa \leq 1 / 2,0<\varepsilon \leq \min \{\kappa, \lambda$, $(1-\lambda)\} / 4$ and every $0<\delta<\kappa-3 \varepsilon$ there exists $c=c(\lambda, \kappa, \delta, \varepsilon)>0$ with the following property: Let $K$ be a symmetric convex body in $\mathbb{R}^{N}$ such that the Euclidean unit ball $B_{2}^{N}$ is the ellipsoid of minimal volume containing $K$. Then for every $\varepsilon^{-1} \leq n \leq \lambda N$ and every $\delta n \leq l \leq(\kappa-2 \varepsilon) n$ the set of all $H \in G_{N, n}$ satisfying

$$
\mathbf{m}\left(P_{H}(K), \kappa\right) \geq c \varphi_{K}(n) W\left(P_{H}(K),\lceil(\kappa-2 \varepsilon) n\rceil, l\right)
$$

has measure larger than or equal to $1-\alpha_{K}(n)$. Moreover,

$$
\begin{aligned}
\int_{G_{N, n}} \mathbf{m}\left(P_{H}(K), \kappa\right) & d \mu_{N, n}(H) \\
& \geq c \varphi_{K}(n) \int_{G_{N, n}} W\left(P_{H}(K),\lceil(\kappa-2 \varepsilon) n\rceil, l\right) d \mu_{N, n}(H) .
\end{aligned}
$$

The next theorem is a version of Theorem 2.3 of [MT4], where the invariant $W\left(P_{H}(K), n / 2, \delta n\right)$ is considered with respect to a slightly more general Euclidean ball. Here we state it for the minimal volume ellipsoid, and with $\kappa n$ replacing $n / 2$. The minimal volume ellipsoid clearly satisfies the assumptions discussed in [MT4] (cf. the comment before (1.5) in [MT4]). The proof for arbitrary $\kappa$ is fully analogous to the proof there.

Theorem 1.4. For every $0<\kappa \leq 1 / 2$ and $0<\delta<\kappa / 2$ there exists $c=c(\kappa, \delta)>0$ with the following property: Let $K \subset \mathbb{R}^{N}$ be a symmetric convex body such that the Euclidean unit ball $B_{2}^{N}$ is the ellipsoid of minimal volume containing $K$. For every $\max \left\{\delta^{-1},(\kappa-2 \delta)^{-1}\right\} \leq n \leq N$, let $m=$ $\lceil(\kappa-2 \delta) n\rceil$. Then the set of all $H \in G_{N, n}$ satisfying

$$
\varphi_{K}(n) W\left(P_{H}(K),\lceil\kappa n\rceil,\lceil\delta n\rceil\right) \geq c \mathbb{E} d_{\mathrm{g}}\left(P_{H}(K), m\right)
$$

has measure larger than or equal to $1-\alpha_{K}(n)$. Moreover there exists $c^{\prime}=$ 
$c^{\prime}(\kappa, \delta)>0$ such that

$$
\varphi_{K}(n) \int_{G_{N, n}} W\left(P_{H}(K),\lceil\kappa n\rceil,\lceil\delta n\rceil\right) d \mu_{N, n}(H) \geq c^{\prime} \mathbb{E} d_{\mathrm{g}}(K, m) .
$$

Despite the fact that the mixing invariant of a body $K$ depends on the Euclidean norm on $\mathbb{R}^{n}$ and therefore depends on the particular position of $K$ it is well known that it provides lower estimates for several structural or symmetry parameters of the Banach space induced by $K$ (cf. [MT3, Sec. 5]). Examples of such parameters are the basis constant, bc $(\cdot)$, the symmetry constant, $\operatorname{sym}(\cdot)$, the factorization constant through a space with a Schauder basis, complexification constants and so on. Theorems 1.1 and 1.3 yield analogous results for all these parameters. Here we shall just state the variant of Theorem 1.1 for the basis and symmetry constants. It is a straightforward consequence of the following known estimates: $\operatorname{bc}(B) \geq(1 / 2) \mathbf{m}(B, 1 / 2)$, $\operatorname{sym}(B) \geq(1 / 4) \mathbf{m}(B, 1 / 20)$ (cf. [MT3, comments after Definition 11 and Theorem 20]).

Corollary 1.5. Let $0<\lambda<1$ and $K \subset \mathbb{R}^{N}$ be as in Theorem 1.1 and let $\eta>0$ be sufficiently small. Let $\eta^{-1} \leq n \leq \lambda N$. Consider the following two sets:

$$
\begin{array}{r}
\left\{H \in G_{N, n} \mid \operatorname{bc}\left(P_{H}(K)\right) \geq c_{1} \mathbb{E} d_{\mathrm{g}}\left(P_{H}(K),\lceil(1 / 2-\eta) n\rceil\right)\right\}, \\
\left\{H \in G_{N, n} \mid \operatorname{sym}\left(P_{H}(K)\right) \geq c_{2} \mathbb{E} d_{\mathrm{g}}\left(P_{H}(K),\lceil(1 / 20-\eta) n\rceil\right)\right\},
\end{array}
$$

where $c_{1}=c(\lambda, 1 / 2, \eta)$ and $c_{2}=c(\lambda, 1 / 20, \eta)$ are as in Theorem 1.1. Then each of these sets has measure larger than or equal to $1-2 \alpha_{K}(n)$.

2. Volumetric invariants and ellipsoids. In this section we shall discuss the behaviour of the invariant $W$. First we consider the case of ellipsoids. In general, an ellipsoid $\mathcal{E}$ in $\mathbb{R}^{n}$ is of the form $\mathcal{E}=u\left(B_{2}^{n}\right)$ for a certain isomorphism $u$ of $\mathbb{R}^{n}$. Let $s_{1}(u) \geq \ldots \geq s_{n}(u)>0$ be a sequence of $s$-numbers of $u$. It easy to see that this sequence does not depend on a particular choice of the isomorphism $u$ and that $\lambda_{i}(\mathcal{E})=s_{i}(u)$ for $i=1, \ldots, n$ are the lengths of the semiaxes of $\mathcal{E}$.

Let $\mathcal{E}$ be an ellipsoid in $\mathbb{R}^{n}$ and $P \in L\left(\mathbb{R}^{n}\right)$ be an orthogonal projection of rank $m$. Then $P(\mathcal{E})$ is an ellipsoid in $F=P\left(\mathbb{R}^{n}\right)$ and we have

$$
\lambda_{i+n-m}(\mathcal{E}) \leq \lambda_{i}(P(\mathcal{E})) \leq \lambda_{i}(\mathcal{E})
$$

for $i=1, \ldots, m$. Indeed, $\lambda_{i}(P(\mathcal{E}))=s_{i}(P u)$ for $i=1, \ldots, m$, where $u$ is any isomorphism of $\mathbb{R}^{n}$ such that $\mathcal{E}=u\left(B_{2}^{n}\right)$. This immediately implies the right hand estimate, and the left hand estimate follows from the formula $s_{i}(T)=\min \{\|T-S\| \mid \operatorname{rank} S<i\}$ for $i=1,2, \ldots$, valid for any operator $T$ on a Hilbert space. 
It is not difficult to show using (2.1) that

$$
W(\mathcal{E}, k, l)=\left(\prod_{i=k-l+1}^{k} \lambda_{i}(\mathcal{E})\right)^{-1 / l}
$$

for any $1 \leq l \leq k \leq n$.

Combining (2.2) and (2.1) we get, for $k \leq m \leq n$,

$$
W(\mathcal{E}, k, l) \leq W(P(\mathcal{E}), k, l) \leq W(\mathcal{E}, k+n-m, l) .
$$

Similarly, for any $E \subset \mathbb{R}^{n}$ with $\operatorname{dim} E=m$, estimates analogous to (2.1) hold for sections $\mathcal{E} \cap E$, and it follows that for $k \leq m \leq n$,

$$
W(\mathcal{E}, k, l) \leq W(\mathcal{E} \cap E, k, l) \leq W(\mathcal{E}, k+n-m, l) .
$$

REMARK. The left and right estimates in both (2.3) and (2.4) are sharp. For the left hand estimates this can be seen by considering the subspaces spanned by the largest $m$ semiaxes of $\mathcal{E}$, and the corresponding projections and sections. For the right hand estimates we consider the subspaces spanned by the smallest $m$ semiaxes of $\mathcal{E}$.

For a general symmetric convex body $B \subset \mathbb{R}^{n}$ the invariant $W(B, k, l)$ is closely related to the same invariant for an $M$-ellipsoid of $B$. This depends on a striking property of such an ellipsoid $\mathcal{E}_{M}$ that volumes of its proportionaldimensional sections and projections are comparable to those of $B$, and this property is also shared by $B+\mathcal{E}_{M}$ and $B \cap \mathcal{E}_{M}$ (where $B+\mathcal{E}_{M}=\{x+y \mid$ $\left.x \in B, y \in \mathcal{E}_{M}\right\}$ is the Minkowski sum). The existence of such ellipsoids (first proved by Milman in [Mi1] and consecutive papers) is highly non-trivial, and they have become a very useful part of the theory by now. Rather than give here the usual definition of an $M$-ellipsoid we shall summarize below its properties needed in this paper. It is well known to specialists that these properties can be easily derived directly from the definition (for example in the form put forward in $[\mathrm{Pi}]$, and in fact are equivalent to this definition). For more information on $M$-ellipsoids we refer the reader to [Mi2] and the references therein, as well as to [Pi] and [MiS2].

FACT 2.1. There exists a numerical constant $C_{0} \geq 1$ such that for every $n \geq 1$ and every symmetric convex body $B \subset \mathbb{R}^{n}$ there exists an ellipsoid $\mathcal{E}_{M}$ (called an $M$-ellipsoid for $B$ ) such that $\left|\mathcal{E}_{M}\right|=|B|$ and:

(i) $\left|B+\mathcal{E}_{M}\right|^{1 / n} \leq C_{0}\left|B \cap \mathcal{E}_{M}\right|^{1 / n}$. In particular, $\left|B+\mathcal{E}_{M}\right|^{1 / n} \leq C_{0}|B|^{1 / n}$ and $\left|B \cap \mathcal{E}_{M}\right|^{1 / n} \geq\left(1 / C_{0}\right)\left|\mathcal{E}_{M}\right|^{1 / n}$.

(ii) For any $0<\lambda<1$ and any subspace $E \subset \mathbb{R}^{n}$ with $l=\operatorname{dim} E=\lambda n$ we have

$$
\begin{aligned}
\left|\left(B+\mathcal{E}_{M}\right) \cap E\right|^{1 / l} & \leq C_{0}^{1 / \lambda}\left|B \cap \mathcal{E}_{M} \cap E\right|^{1 / l} \\
\left|P_{E}\left(B+\mathcal{E}_{M}\right)\right|^{1 / l} & \leq C_{0}^{1 / \lambda}\left|P_{E}\left(B \cap \mathcal{E}_{M}\right)\right|^{1 / l} .
\end{aligned}
$$


As a consequence one has

Corollary 2.2. Let $B \subset \mathbb{R}^{n}$ be a symmetric convex body. Let $\mathcal{E}_{M}$ be an $M$-ellipsoid for $B$ and let $C_{0}$ be as in Fact 2.1. Let $0<\delta<1$. For every $\delta n \leq l \leq k \leq n$ we have:

(i) Set $B_{1}=B, B_{2}=\mathcal{E}_{M}, B_{3}=B+\mathcal{E}_{M}$ and $B_{4}=B \cap \mathcal{E}_{M}$. Then for all $i, j=1, \ldots, 4$ we have

$$
W\left(B_{i}, k, l\right) \leq C_{0}^{1 / \delta} W\left(B_{j}, k, l\right) .
$$

(ii) For every $k \leq m \leq n$ and $E \subset \mathbb{R}^{n}$ with $\operatorname{dim} E=m$, we have

$$
C_{0}^{-2 / \delta} W(B, k, l) \leq W\left(P_{E}(B), k, l\right) \leq C_{0}^{2 / \delta} W(B, k+n-m, l) .
$$

Condition (i) follows from Fact 2.1, while (ii) follows from (i) and (2.3).

Besides the invariant $W$ considered up to now, one can define some other related volumetric invariants. For example, one such invariant arises naturally in arguments in the last two sections which contain the technical kernel of this paper. Namely, we consider an invariant similar to $W(\cdot, \cdot, \cdot)$ but based on projections rather than sections, that is,

$$
\widetilde{W}(B, k, l)=\inf _{E} \sup _{F}\left(\frac{\left|B_{2}^{l}\right|}{\left|P_{F}(B)\right|}\right)^{1 / l},
$$

where the infimum is taken over all $k$-dimensional subspaces $E \subset \mathbb{R}^{n}$ and the supremum is taken over all $l$-dimensional subspaces $F \subset E$.

Clearly, $\widetilde{W}(B, k, l) \leq W(B, k, l)$. Much more is in fact true: these invariants are well equivalent on proportional levels. Indeed, if $\mathcal{E}$ is an ellipsoid, the same argument as in (2.2) yields the same formula for $\widetilde{W}(\mathcal{E}, k, l)$, and hence $\widetilde{W}(\mathcal{E}, k, l)=W(\mathcal{E}, k, l)$. Corollary 2.2 says in particular that on proportional levels the invariants $W(B, \cdot, \cdot)$ and $W\left(\mathcal{E}_{M}, \cdot, \cdot\right)$ are well comparable. The same holds for the invariant $\widetilde{W}(\cdot, \cdot, \cdot)$. Therefore for all $\delta n \leq l \leq k \leq n$ we have

$$
C_{0}^{-2 / \delta} W(B, k, l) \leq \widetilde{W}(B, k, l) \leq W(B, k, l) .
$$

Also, the same holds for invariants of "mixed type" involving both sections and projections as considered in [MT1] (cf. also [MT3, Section 7]).

We illustrate this circle of ideas by the following proposition which is a strengthening, in a sense, of an analogous result from [MT4, Proposition 3.2]. It can be proved directly by geometric considerations similar to Propositions 4.1 and 3.2 from [MT4]; however, we provide here a formal argument based on (2.6). It is not clear whether the assumption $\alpha \leq 1 / 2$ below is necessary.

Proposition 2.3. For every $0<\alpha \leq 1 / 2$ and $0<\delta<\alpha / 4$ there exist $c_{1}=c_{1}(\alpha, \delta)>0$ and $c_{2}=c_{2}(\alpha, \delta)>0$ such that for every symmetric 
convex body $B \subset \mathbb{R}^{n}$ with $P_{E}(B) \supset P_{E}\left(B_{2}^{n}\right)$ for some $k$-dimensional subspace $E \subset \mathbb{R}^{n}$ with $k=\lceil\alpha n\rceil-1$, the measure of the set

$$
\left\{H \in G_{n, k-3\lceil\delta n\rceil} \mid P_{H}(B) \supset c_{1} P_{H}\left(B_{2}^{n}\right)\right\}
$$

is greater than or equal to $1-e^{-c_{2} n}$.

Proof. Let $l=\lceil\delta n\rceil$. Then $P_{E}(B) \supset P_{E}\left(B_{2}^{n}\right)$ yields $\widetilde{W}(B, k, l) \leq 1$. Thus, by $(2.6)$, we get $W(B, k, l) \leq C_{0}^{2 / \delta}$. Let $F \subset E$ be the linear subspace spanned by the largest $2 k$ semiaxes of $\mathcal{E}$, where $\mathcal{E}$ is an $M$-ellipsoid for $B$. By the remark following (2.4) we have $W(\mathcal{E}, k, l)=W(\mathcal{E} \cap F, k, l)$. By Corollary $2.2(\mathrm{i})$ we get $W(B, k, l) \geq C_{0}^{-2 / \delta} W(\widetilde{B}, k, l)$, where $\widetilde{B}=B \cap F$. Thus $W(\widetilde{B}, k, l) \leq C_{0}^{4 / \delta}$. The proof is completed by applying first Lemma 4.2 from $[\mathrm{MT} 4$ ] to $\widetilde{B}$ (note that $k=\lceil\alpha n\rceil=(\operatorname{dim} F) / 2$ ) and then using Proposition 3.2 therein.

3. Gaussian version of Theorem 1.3. We denote by $\gamma$ a standard $N(0,1)$ distributed (real-valued) Gaussian random variable. For an $N$-dimensional Hilbert space $H$, by a normalized Gaussian vector $g: \Omega \rightarrow H$ we mean a random vector of the form $g=N^{-1 / 2} \sum_{i=1}^{N} \gamma_{i} u_{i}$, where the $\gamma_{i}$ are independent copies of $\gamma$, and $\left\{u_{i}\right\}$ is any orthonormal basis in $H$. Thus $g$ has distribution $N\left(0, N^{-1} I_{H}\right)$ (where $I_{H}$ denotes the identity matrix on $H$ ). Its density is equal to $(N / 2 \pi)^{N / 2} \exp \left(-N\|x\|_{2}^{2} / 2\right)$ and is clearly rotation invariant.

Let us recall the following basic fact (cf. e.g. [MT3] where it is used in a similar context).

FACT 3.1. Let $g$ be a normalized Gaussian vector in an $N$-dimensional Hilbert space $H$. Then

(i) For every $k$-dimensional subspace $E \subset H, \sqrt{N / k} P_{E} g$ is a normalized Gaussian vector in $E$.

(ii) For every orthogonal pair of subspaces $E_{1}, E_{2} \subset H$ the random vectors $P_{E_{1}} g$ and $P_{E_{2}} g$ are independent.

(iii) For every Borel set $B \subset H$ we have

$$
\mathbb{P}\{\omega \in \boldsymbol{\Omega} \mid g(\omega) \in B\} \leq e^{N / 2}|B| /\left|B_{2}^{N}\right| .
$$

Now we pass to a description of Gaussian matrices. For $n, N \geq 1$, let $\Gamma_{n, N}=\Gamma_{n, N, \omega}$ be an $n \times N$ random matrix with independent $N(0,1 / N)$ distributed entries. We shall treat $\Gamma_{n, N}$ as a linear random operator from $\mathbb{R}^{N}$ to $\mathbb{R}^{n}$. The following fact is well known (cf. [MaS, Fact 3.2 and the comments afterwards]).

FACT 3.2. Let $0<\lambda<1$ and $A>0$. There exist $C=C(A)>0$ and $c=c(A, \lambda)>0$ such that for every $1 \leq n \leq \lambda N$, letting $E_{\omega}=\left(\operatorname{ker} \Gamma_{n, N, \omega}\right)^{\perp}$ 
for $\omega \in \boldsymbol{\Omega}$, we have

$$
\begin{aligned}
\mathbb{P}\left\{\omega \in \Omega \mid c\|x\|_{2} \leq\left\|\Gamma_{n, N, \omega} x\right\|_{2} \leq C\|x\|_{2} \text { for every } x\right. & \left.\in E_{\omega}\right\} \\
& \geq 1-e^{-A N} .
\end{aligned}
$$

For $A>0$ and $0<\lambda<1$ let $\boldsymbol{\Omega}^{\prime}=\boldsymbol{\Omega}^{\prime}(A, \lambda)$ be the subset of $\boldsymbol{\Omega}$ from Fact 3.2. The definition of $\boldsymbol{\Omega}^{\prime}$ easily implies that for $\omega \in \boldsymbol{\Omega}^{\prime}$ we have

$$
c W\left(\Gamma_{n, N, \omega}(K), k, l\right) \leq W\left(P_{E_{\omega}}(K), k, l\right) \leq C W\left(\Gamma_{n, N, \omega}(K), k, l\right)
$$

and

$$
(c / C) \mathbf{m}\left(\Gamma_{n, N, \omega}(K), \kappa\right) \leq \mathbf{m}\left(P_{E_{\omega}}(K), \kappa\right) \leq(C / c) \mathbf{m}\left(\Gamma_{n, N, \omega}(K), \kappa\right),
$$

where $C, c>0$ are from Fact 3.2. Indeed, observe that $\Gamma_{n, N, \omega}=\Gamma_{n, N, \omega} P_{E_{\omega}}$, and set $S_{\omega}=\Gamma_{n, N, \omega} \mid E_{\omega}$. Thus $S_{\omega}$ is a good isomorphism for $\omega \in \Omega^{\prime}$. In particular it allows a good control of the volumes on every subspace, which in turn implies (3.2). Furthermore, $S_{\omega}$ is an isometry from $\left(E_{\omega}, P_{E_{\omega}}(K)\right)$ onto $\left(\mathbb{R}^{n}, \Gamma_{n, N, \omega}(K)\right)$ and hence for every $T \in L\left(E_{\omega}\right)$ we have $\|T\|_{P_{E_{\omega}}(K)}=$ $\left\|S_{\omega} T S_{\omega}^{-1}\right\|_{\Gamma_{n, N, \omega}(K)}$. Clearly $T \in \operatorname{Mix}_{E_{\omega}}(\kappa, 1)$ implies that $(C / c) S_{\omega} T S_{\omega}^{-1} \in$ $\operatorname{Mix}_{n}(\kappa, 1)$ for every $T \in L\left(E_{\omega}\right)$. This easily yields (3.3).

For an arbitrary Borel subset $\mathcal{F} \subset G_{N, n}$ let

$$
\Omega(\mathcal{F})=\left\{\omega \in \boldsymbol{\Omega} \mid\left(\operatorname{ker} \Gamma_{n, N, \omega}\right)^{\perp} \in \mathcal{F}\right\} .
$$

The rotation invariance of the distribution of Gaussian matrices implies that $\mathbb{P}(\Omega(\mathcal{F}))=\mu_{N, n}(\mathcal{F})$. Therefore for any subset $\boldsymbol{\Omega}_{1} \subset \boldsymbol{\Omega}$ we have

$$
\mu_{N, n}\left(\left\{E \in G_{N, n} \mid E=\left(\operatorname{ker} \Gamma_{n, N, \omega}\right)^{\perp} \text { for some } \omega \in \boldsymbol{\Omega}_{1}\right\}\right) \geq \mathbb{P}\left(\boldsymbol{\Omega}_{1}\right) .
$$

We shall also need the following tail estimates for the operator norm and the determinant of a Gaussian square matrix (cf. e.g. [MT4, Facts 1.4(i) and 1.5]).

FACT 3.3. (i) For every $A \geq 1$ there is $C=C(A)>1$ such that for all $N \geq 1$

$$
\mathbb{P}\left\{\omega \in \boldsymbol{\Omega} \mid\left\|\Gamma_{N, N, \omega}\right\|_{2} \leq C\right\}>1-e^{-A N} .
$$

(ii) For every $A \geq 1$ there is $\varrho=\varrho(A)>0$ such that for all $N \geq 4$,

$$
\mathbb{P}\left\{\omega \in \boldsymbol{\Omega}|| \operatorname{det} \Gamma_{N, N, \omega} \mid \geq \varrho^{N}\right\}>1-e^{-A N} .
$$

Moreover, we may take $\varrho=c e^{-2 A}$, where $c>0$ is a suitable numerical constant.

The following theorem is a Gaussian version of Theorem 1.3.

TheOrem 3.4. For every $0<\lambda<1,0<\kappa \leq 1 / 2,0<\varepsilon \leq \min \{\kappa, \lambda$, $(1-\lambda)\} / 4$ and every $0<\delta<\kappa-2 \varepsilon$ there exists $c=c(\lambda, \kappa, \delta, \varepsilon)>0$ with the following property: Let $K$ be a symmetric convex body in $\mathbb{R}^{N}$ such that the Euclidean unit ball $B_{2}^{N}$ is the ellipsoid of minimal volume containing $K$. 
Then for every $\max \left\{\varepsilon^{-1}, k^{*}(K)\right\} \leq n \leq \lambda N$ and every $\delta n \leq l \leq(\kappa-2 \varepsilon) n$ we have

$$
\begin{array}{r}
\mathbb{P}\left\{\omega \in \boldsymbol{\Omega} \mid \mathbf{m}\left(\Gamma_{n, N, \omega}(K), \kappa\right) \geq c \sqrt{n / N} W\left(\Gamma_{n, N, \omega}(K),\lceil(\kappa-2 \varepsilon) n\rceil, l\right)\right\} \\
\\
\geq 1-\alpha_{K}(n) / 2 .
\end{array}
$$

Assuming the truth of Theorem 3.4 we complete the proof of Theorem 1.3 as follows.

Proof of Theorem 1.3. First note that it is enough to consider only the case when $n \geq k^{*}(K)$. The remaining case is a direct consequence of the definition of $k^{*}(K)$ and (1.13) and (1.9) (note that $a=1$ for the ellipsoid of minimal volume). Let $\boldsymbol{\Omega}^{\prime}$ be the subset of $\boldsymbol{\Omega}$ from Fact 3.2 for $A=4$ and $\lambda$, and let $\boldsymbol{\Omega}^{\prime \prime}$ be the subset of $\boldsymbol{\Omega}$ considered in Theorem 3.4. Combining (3.2) and (3.3) we infer that for $\omega \in \boldsymbol{\Omega}_{1}=\boldsymbol{\Omega}^{\prime} \cap \boldsymbol{\Omega}^{\prime \prime}$ we have

$$
\mathbf{m}\left(P_{E_{\omega}}(K), \kappa\right) \geq c^{\prime} \varphi_{K}(n) W\left(P_{E_{\omega}}(K),\lceil(\kappa-2 \varepsilon) n\rceil, l\right),
$$

where $c^{\prime}=c^{\prime}(\lambda, \kappa, \delta, \varepsilon)$ depends on $\lambda, \kappa, \delta$ and $\varepsilon$ only. The proof of (1.14) is completed by observing that by the definition of $\boldsymbol{\Omega}_{1}$ and by (3.4) the Haar measure of the subset considered in (1.14) is greater than or equal to $1-\alpha_{K}(n) / 2-e^{-4 n} \geq 1-\alpha_{K}(n)$.

To prove the integral part of the theorem note that by (3.5) we have

$$
\begin{aligned}
\int_{G_{N, n}} \mathbf{m}\left(P_{H}(K), \kappa\right) d \mu_{N, n}(H) & \\
& \geq c^{\prime} \varphi_{K}(n) \int_{\mathcal{A}} W\left(P_{H}(K),\lceil(\kappa-2 \varepsilon) n\rceil, l\right) d \mu_{N, n}(H),
\end{aligned}
$$

where $\mathcal{A}$ is the subset of $G_{N, n}$ on which the inequality (3.5) is satisfied. Also

$$
\begin{aligned}
\int_{G_{N, n}} \mathbf{m}\left(P_{H}(K), \kappa\right) d \mu_{N, n}(H) & \\
& \geq c^{\prime \prime} \varphi_{K}(n) \int_{G_{N, n} \backslash \mathcal{A}} W\left(P_{H}(K),\lceil(\kappa-2 \varepsilon) n\rceil, l\right) d \mu_{N, n}(H)
\end{aligned}
$$

for a suitable numerical constant $c^{\prime \prime}>0$. Indeed, since

$$
N^{-1 / 2} B_{2}^{N} \subset K
$$

we have $W\left(P_{H}(K),\lceil(\kappa-2 \varepsilon) n\rceil, l\right) \leq N^{1 / 2}$ for every $H \in G_{N, n}$. Thus the integral on the right hand side of (3.7) is less than or equal to $\alpha_{K}(n) \sqrt{N}$. On the other hand, by the well known fact on shrinking of diameter of random projections of a symmetric convex body (which follows from Milman's proof of Dvoretzky's theorem; cf. [MiS1, Th. 4.2], see also [MT4, Proposition 4.3]), there is a numerical constant $C>0$ such that the inclusion

$$
P_{H}(K) \subset C(n / N)^{1 / 2} P_{H}\left(B_{2}^{N}\right)
$$


holds on a subset $\mathcal{H}$ of $G_{N, n}$ with measure close to 1 . Thus, by (3.8), (3.9) and (1.3), for $H \in \mathcal{H}$ we have $\mathbf{m}\left(P_{H}(K), \kappa\right) \geq 1 / C \sqrt{n}$. Hence (3.7) follows by the definitions of $\varphi_{K}(n)$ and $\alpha_{K}(n)$.

Finally, the integral inequality in Theorem 1.3 , with $c=\min \left\{c^{\prime}, c^{\prime \prime}\right\} / 2$, follows by adding (3.6) and (3.7).

A well known strategy of proving results like Theorem 3.4 is based on two steps. The first is to provide an estimate for the probability "for a single operator", and the second is a continuity argument based on finding an appropriate net in the set of operators considered and using the previous probability estimate. We present this argument in two separate sections which follow.

4. Estimate for a single operator. A new technical point of Theorem 3.4 compared with a finite-dimensional result from [MT2] is made possible by an effective use of the geometry of contact points between a convex body and its John ellipsoid. This idea was already used in a similar way in [MT4].

We shall need the following version of Proposition 5.2 of [MT4].

Proposition 4.1. Let $n, m \geq 1$ and $B \subset \mathbb{R}^{n}$ be a symmetric convex body. Let $h_{1}, \ldots, h_{m}$ be independent normalized Gaussian vectors in $\mathbb{R}^{n}$. For $i=1, \ldots, m$ consider random vectors

$$
y_{i}=\sum_{j=1}^{i} b_{i, j} h_{j}
$$

with $b_{i, i}>0$ for $i=1, \ldots, m$. Let $1 \leq k \leq n$, and let $S \in L\left(\mathbb{R}^{n}\right)$ be an operator such that for some $k$-dimensional subspace $E \subset \mathbb{R}^{n}$ we have $\|S x\|_{2} \geq\|x\|_{2}$ for every $x \in E$. Let $w_{i} \in \mathbb{R}^{n}$ for $i=1, \ldots, m$. Then for any $1 \leq l \leq k$,

$$
\mathbb{P}\left\{w_{i}+S y_{i} \in B \text { for } i=1, \ldots, m\right\} \leq\left(\prod_{j=1}^{m} b_{j, j}\right)^{-l}(e n / l)^{l m / 2}(\widetilde{W}(B, k, l))^{-l m},
$$

where $\widetilde{W}(B, k, l)$ is defined in $(2.5)$.

If the vectors $w_{i}$ belong to the range of $S$ then the proposition follows from Proposition 5.2 of [MT4]. The general case treated here needs a modification of the argument applied there, with the vectors $w_{i}$ replacing $S z_{i}$. This, in turn, requires the use of the invariant $\widetilde{W}$ instead of $W$. We leave the further details to the reader.

Let us recall some basic facts concerning the ellipsoid of minimal volume. Let $K \subset \mathbb{R}^{N}$ be a symmetric convex body such that $B_{2}^{N}$ is the ellipsoid of minimal volume containing $B$. A classical result by Dvoretzky and Rogers 
says that there exist contact points $x_{1}, \ldots, x_{N}$ (that is, $\left\|x_{i}\right\|_{K}=\left\|x_{i}\right\|_{2}=1$ ) and orthonormal vectors $u_{1}, \ldots, u_{N}$ in $\left(\mathbb{R}^{N},\|\cdot\|_{2}\right)$ such that

$$
x_{i}=\sum_{j=1}^{i} b_{i, j} u_{j} \quad \text { and } \quad b_{i, i} \geq((N-i+1) / N)^{1 / 2}
$$

for $i=1, \ldots, N$ (cf. e.g. [T, $\S 15]$ ). By rotating the body $K$ if necessary, we may assume that $u_{j}=e_{j}$, where $\left\{e_{j}\right\}$ is the standard unit vector basis in $\mathbb{R}^{N}$.

Let $\lambda, \delta, \kappa, \varepsilon$ and $K$ be as in Theorem 3.4 and let $x_{1}, \ldots, x_{N}$ be contact points of $K$ satisfying (4.1). Fix $n$ as in Theorem 3.4. For $i=1, \ldots, N$ consider random Gaussian vectors in $\mathbb{R}^{n}$ defined by $g_{i}=\Gamma_{n, N} e_{i}$, and set

$$
y_{i}=\Gamma_{n, N} x_{i}=\sum_{j=1}^{i} b_{i, j} \Gamma_{n, N} e_{j}=\sum_{j=1}^{i} b_{i, j} g_{j} .
$$

Let $\sigma=\{n+1, n+2, \ldots, n+[\varepsilon n]\}$, where $[\varepsilon n]$ denotes the largest integer smaller than or equal to $\varepsilon n$. Given a mixing operator $T$ we want to estimate the probability of the set of $\omega \in \boldsymbol{\Omega}$ such that

$$
T y_{i} \in c \Gamma_{n, N, \omega}(K) \quad \text { for } i \in \sigma,
$$

for some $c>0$. Proposition 4.1 provides an estimate of this type when the convex set on the right hand side is fixed. In our case, when the set depends on $\omega$ we will replace (4.3) with a stronger condition of the form $S h_{i}\left(\omega_{2}\right) \in B\left(\omega_{1}\right)$ with $B\left(\omega_{1}\right)$ and $h_{i}\left(\omega_{2}\right)$ being independent. This will require several notations.

Let $Q$ be the random orthogonal projection in $\mathbb{R}^{n}$ with $\operatorname{ker} Q=\operatorname{span}\left\{g_{i} \mid\right.$ $i \in \sigma\}$. Note that for $\omega \in \boldsymbol{\Omega}$,

$$
Q \Gamma_{n, N}=Q \Gamma_{n, N} \widetilde{P}
$$

where $\widetilde{P}$ is the orthogonal projection in $\mathbb{R}^{N}$ with ker $\widetilde{P}=\operatorname{span}\left\{e_{i} \mid i \in \sigma\right\}$.

To make the argument more transparent, we shall assume as we may that

$$
(\boldsymbol{\Omega}, \mathbb{P})=\left(\Omega_{1}, \mathbb{P}_{1}\right) \times\left(\Omega_{2}, \mathbb{P}_{2}\right)
$$

and we shall write $\omega=\left(\omega_{1}, \omega_{2}\right)$. Moreover, we shall assume that the $g_{i}(\omega)$ for $i \in \sigma$ depend only on $\omega_{2}$ while the remaining $g_{i}(\omega)$ 's depend only on $\omega_{1}$. In particular, by (4.4) we have

$$
Q \Gamma_{n, N, \omega}(K)=Q \Gamma_{n, N, \omega} \widetilde{P}(K),
$$

and since $\widetilde{P}(K) \subset \operatorname{span}\left\{e_{i} \mid i \leq N\right.$ and $\left.i \notin \sigma\right\}$ we infer that the set

$$
B\left(\omega_{1}\right)=\Gamma_{n, N, \omega} \widetilde{P}(K) \subset \mathbb{R}^{n}
$$

depends only on $\omega_{1}$ (which is the first step in the direction described above). Let $\mathcal{E}\left(\omega_{1}\right) \subset \mathbb{R}^{n}$ be an $M$-ellipsoid for $B\left(\omega_{1}\right)$. (The use of the $M$-ellipsoid is 
required in the last section to control the cardinality of nets in the class of mixing operators.) Finally, let

$$
\begin{aligned}
& \bar{B}\left(\omega_{1}\right)=B\left(\omega_{1}\right)+\mathcal{E}\left(\omega_{1}\right) \subset \mathbb{R}^{n}, \\
& \widetilde{B}\left(\omega_{1}\right)=Q \bar{B}\left(\omega_{1}\right) \subset Q \mathbb{R}^{n},
\end{aligned}
$$

and note that both $\bar{B}\left(\omega_{1}\right)$ and $\widetilde{B}\left(\omega_{1}\right)$ depend only on $\omega_{1}$, i.e., do not depend on $\omega_{2}$.

Let $\Omega_{0}$ be the subset of $\boldsymbol{\Omega}$ consisting of all $\omega$ such that

$$
\begin{aligned}
\left|\operatorname{det} \Gamma_{n, N, \omega}\right| \mathbb{R}^{n} \mid & \geq(\varrho \sqrt{n / N})^{n}, \\
\left\|\Gamma_{n, N, \omega} \mid \mathbb{R}^{n+[\varepsilon n]}\right\|_{2} & \leq C \sqrt{(1+\varepsilon) n / N},
\end{aligned}
$$

where both $\varrho$ and $C$ are taken from Fact 3.3 for $A=4$. Since the normalization of a random matrix $\Gamma_{n, N}$ depends on $N$, in order to use Fact 3.3 to restrictions of the matrices above, a suitable normalization is required. After such a normalization we get

$$
\mathbb{P}\left(\boldsymbol{\Omega}_{0}\right) \geq 1-2 e^{-4 n} .
$$

Set

$$
\Omega_{1}^{\prime}=\left\{\omega_{1} \in \Omega_{1} \mid\left(\omega_{1}, \omega_{2}\right) \in \Omega_{0} \text { for some } \omega_{2} \in \Omega_{2}\right\} .
$$

We shall use the following obvious properties of $\boldsymbol{\Omega}_{0}$. Since the matrix in (4.9) depends only on $\omega_{1}$, it follows that if $\omega_{1} \in \Omega_{1}^{\prime}$ then for every $\bar{\omega}_{2} \in \Omega_{2}$,

$$
\left|\operatorname{det}\left[g_{i}(\bar{\omega})\right]_{i=1}^{n}\right| \geq(\varrho \sqrt{n / N})^{n},
$$

where $\bar{\omega}=\left(\omega_{1}, \bar{\omega}_{2}\right)$. For every $\omega \in \Omega_{0}$ and every $i=1, \ldots, n+[\varepsilon n]$,

$$
\left\|\Gamma_{n, N, \omega} x_{i}\right\|_{2} \leq 2 C \sqrt{n / N} .
$$

Till the end of the paper, in order to avoid tedious considerations, we shall assume that both $\kappa n$ and $\varepsilon n$ are integers. The general case follows the same line of argument.

In the notation above we have

Lemma 4.2. Let $\lambda, \delta, \kappa, \varepsilon, n, l$ and $K$ be as in Theorem 3.4. There exists $C_{1}=C_{1}(\lambda, \delta, \kappa, \varepsilon)$ such that for every $\omega_{1} \in \Omega_{1}$, every $\alpha>0$ and every operator $T \in \operatorname{Mix}_{n}((\kappa-\varepsilon) n, 1)$ one has

$$
\mathbb{P}_{2}\left\{\omega_{2} \in \Omega_{2} \mid Q T y_{i} \in A(\alpha) \widetilde{B}\left(\omega_{1}\right) \text { for } i \in \sigma,\left(\omega_{1}, \omega_{2}\right) \in \Omega_{0}\right\}<\left(C_{1} \alpha\right)^{\varepsilon l n},
$$
where

$$
A(\alpha)=\alpha \sqrt{n / N} W\left(\widetilde{B}\left(\omega_{1}\right),(\kappa-2 \varepsilon) n, l\right) .
$$

Proof. Fix $T \in \operatorname{Mix}_{n}((\kappa-\varepsilon) n, 1)$ and let $E$ be a linear subspace in $\mathbb{R}^{n}$ with $\operatorname{dim} E=(\kappa-\varepsilon) n$ such that

$$
\left\|P_{E^{\perp}} T x\right\|_{2} \geq\|x\|_{2} \quad \text { for every } x \in E \text {. }
$$


For $i \in \sigma$ set $h_{i}^{\prime}\left(\omega_{2}\right)=P_{E} g_{i}\left(\omega_{2}\right)$ and $h_{i}^{\prime \prime}\left(\omega_{2}\right)=P_{E^{\perp}} g_{i}\left(\omega_{2}\right)$. Thus $g_{i}\left(\omega_{2}\right)=$ $h_{i}^{\prime}\left(\omega_{2}\right)+h_{i}^{\prime \prime}\left(\omega_{2}\right)$. Since by Fact $3.1(\mathrm{ii}), h_{i}^{\prime}\left(\omega_{2}\right)$ and $h_{i}^{\prime \prime}\left(\omega_{2}\right)$ are independent Gaussian vectors we may assume that $\left(\Omega_{2}, \mathbb{P}_{2}\right)=\left(\Omega_{2}^{\prime}, \mathbb{P}_{2}^{\prime}\right) \times\left(\Omega_{2}^{\prime \prime}, \mathbb{P}_{2}^{\prime \prime}\right)$ and that for every $i \in \sigma, h_{i}^{\prime}$ depends only on $\omega_{2}^{\prime}$, while $h_{i}^{\prime \prime}$ depends only on $\omega_{2}^{\prime \prime}$. Therefore we may write $h_{i}^{\prime}\left(\omega_{2}^{\prime}\right)$ (resp., $\left.h_{i}^{\prime \prime}\left(\omega_{2}^{\prime \prime}\right)\right)$ instead of $h_{i}^{\prime}\left(\omega_{2}\right)$ (resp., $\left.h_{i}^{\prime \prime}\left(\omega_{2}\right)\right)$. Note that since $g_{i}$ 's for $i=1, \ldots, N$ are independent, the collection of vectors $\left\{h_{i}^{\prime}, h_{j}^{\prime \prime} \mid i, j \in \sigma\right\}$ is independent as well.

Note that the projection $Q$ depends on $\omega_{2}=\left(\omega_{2}^{\prime}, \omega_{2}^{\prime \prime}\right)$. However, in order to apply Proposition 4.1 we need the dependence on $\omega_{2}^{\prime \prime}$ only. This is achieved by composing with one more orthogonal projection. Fix $\omega_{2}^{\prime \prime}$ (and therefore the vectors $\left.h_{i}^{\prime \prime}\left(\omega_{2}^{\prime \prime}\right)\right)$. Since $\operatorname{dim} P_{E^{\perp}} T(E)=(\kappa-\varepsilon) n$ there exists a subspace $F \subset P_{E^{\perp}} T(E)$ with $\operatorname{dim} F=(\kappa-2 \varepsilon) n$ orthogonal to $\operatorname{span}\left\{h_{i}^{\prime \prime} \mid i \in \sigma\right\}$. Note that $F \subset E^{\perp}$ is orthogonal to $\operatorname{span}\left\{h_{i}^{\prime}\left(\omega_{2}^{\prime}\right) \mid i \in \sigma\right\} \subset E$ for every $\omega_{2}^{\prime} \in \Omega_{2}^{\prime}$ as well. Hence $F$ is orthogonal to $\operatorname{ker} Q$ and we have $P_{F} Q=P_{F}$.

Define the operator

$$
S=P_{F} T \mid E: E \rightarrow P_{E^{\perp}} T(E) .
$$

By (4.16) and (4.17) there exists a subspace $E_{0} \subset E$ with $\operatorname{dim} E_{0}=(\kappa-2 \varepsilon) n$ such that

$$
\|S x\|_{2} \geq\|x\|_{2} \quad \text { for } x \in E_{0} .
$$

Since $\omega_{1}$ is fixed and $g_{j}$ 's for $j=1, \ldots, n$ depend only on $\omega_{1}$, and $g_{j}$ 's for $j \in \sigma$ depend only on $\omega_{2}$, using the fact that $\omega_{2}^{\prime \prime}$ is fixed, by (4.2), for every $i \in \sigma$, we can write

$$
y_{i}=\bar{w}_{i}+\sum_{j=n+1}^{i} b_{i, j} h_{j}^{\prime}\left(\omega_{2}^{\prime}\right)
$$

where

$$
\bar{w}_{i}=\sum_{j=1}^{n} b_{i, j} g_{j}\left(\omega_{1}\right)+\sum_{j=n+1}^{i} b_{i, j} h_{j}^{\prime \prime}\left(\omega_{2}^{\prime \prime}\right) .
$$

Thus for each $i \in \sigma$ by (4.19) we have

$$
P_{F} T y_{i}=w_{i}+P_{F} T\left(\sum_{j=n+1}^{i} b_{i, j} h_{j}^{\prime}\left(\omega_{2}^{\prime}\right)\right)
$$

where $w_{i}=P_{F} T \bar{w}_{i}$.

Since $P_{F}=P_{F} Q$, by (4.20) and (4.17), we have

$$
\begin{aligned}
& \quad \mathbb{P}_{2}^{\prime}\left\{\omega_{2}^{\prime} \in \Omega_{2}^{\prime} \mid Q T y_{i} \in A(\alpha) \widetilde{B}\left(\omega_{1}\right) \text { for } i \in \sigma \text { and }\left(\omega_{1}, \omega_{2}\right) \in \Omega_{0}\right\} \\
& \leq \mathbb{P}_{2}^{\prime}\left\{\omega_{2}^{\prime} \in \Omega_{2}^{\prime} \mid P_{F} T y_{i} \in A(\alpha) P_{F}\left(\widetilde{B}\left(\omega_{1}\right)\right) \text { for every } i \in \sigma\right\}
\end{aligned}
$$$$
=\mathbb{P}_{2}^{\prime}\left\{\omega_{2}^{\prime} \in \Omega_{2}^{\prime} \mid w_{i}+S\left(\sum_{j=n+1}^{i} b_{i, j} h_{j}^{\prime}\right) \in A(\alpha) P_{F}\left(\widetilde{B}\left(\omega_{1}\right)\right) \text { for every } i \in \sigma\right\} \text {. }
$$ 
Now, Proposition 4.1 applied to $h_{i}=\sqrt{N /(\kappa-\varepsilon) n} h_{i}^{\prime}\left(\omega_{2}^{\prime}\right)$, the operator $S: E \rightarrow F$ and $B=A(\alpha) \sqrt{N /(\kappa-\varepsilon) n} P_{F}\left(\widetilde{B}\left(\omega_{1}\right)\right)$ shows that the latter probability is less than or equal to

$$
\begin{aligned}
A(\alpha)^{\varepsilon l n} & (N /(\kappa-\varepsilon) n)^{\varepsilon l n / 2} \\
& \times\left(\prod_{j=n+1}^{n+\varepsilon n} b_{j, j}\right)^{-l}(e n / l)^{\varepsilon l n / 2} \widetilde{W}\left(P_{F}\left(\widetilde{B}\left(\omega_{1}\right)\right),(\kappa-2 \varepsilon) n, l\right)^{-\varepsilon l n}
\end{aligned}
$$

(note that the invariant $\widetilde{W}(\cdot, \cdot, \cdot)$ is homogeneous of degree -1 , so

$$
\widetilde{W}\left(\varrho P_{F}\left(\widetilde{B}\left(\omega_{1}\right)\right), k, l\right)=\varrho^{-1} \widetilde{W}\left(P_{F}\left(\widetilde{B}\left(\omega_{1}\right)\right), k, l\right)
$$

for every $\varrho>0)$. By (4.1), since $n+\varepsilon n \leq(1+3 \lambda) N / 4$ we deduce that $\prod_{j=n+1}^{n+\varepsilon n} b_{j, j} \geq c^{\varepsilon n}$ for suitable $c=c(\lambda, \kappa, \varepsilon)$. Thus by (2.6), Corollary 2.2(ii) and the definition of $A(\alpha)$ we infer that the right hand side of (4.21) is less than or equal to $\left(C_{1} \alpha\right)^{\varepsilon l n}$, where $C_{1}=C_{1}(\lambda, \kappa, \delta, \varepsilon)$ depends on $\lambda, \kappa, \delta$ and $\varepsilon$ only. The proof of the lemma is completed by combining this estimate with (4.21) and (4.22) and integrating the resulting inequality with respect to $\omega_{2}^{\prime \prime}$.

5. The $\varepsilon$-net argument. For every fixed $\omega_{1} \in \Omega_{1}$ and every $\alpha>0$ let

$$
\widetilde{\mathbf{A}}_{\alpha}=\left\{T \in L\left(\mathbb{R}^{n}\right) \mid T y_{i}(\omega) \in \alpha \bar{B}\left(\omega_{1}\right) \text { for every } i=1, \ldots, n\right\},
$$

where $y_{i}(\omega)$ 's and $\bar{B}\left(\omega_{1}\right)$ are defined in (4.2) and (4.7) respectively. Set

$$
\mathbf{A}_{\alpha}=\widetilde{\mathbf{A}}_{\alpha} \cap \operatorname{Mix}_{n}((\kappa-2 \varepsilon) n, 1) .
$$

Observe that each $y_{j}$ for $j=1, \ldots, n$ depends on $g_{i}\left(\omega_{1}\right)$ for $i=1, \ldots, j$ only. Thus $\widetilde{\mathbf{A}}_{\alpha}$ and therefore $\mathbf{A}_{\alpha}$ depend on $\omega_{1}$ only. Recall that $\Omega_{1}^{\prime}$ is defined in (4.12).

Lemma 5.1. Let $\lambda, \kappa, \delta, \varepsilon$ and $K$ be as in Theorem 3.4. For every $\omega_{1} \in \Omega_{1}^{\prime}$ and every $\alpha>0$ the set $\mathbf{A}_{\alpha}$ of operators admits a $\left(1 / 4 C_{1}\right) \sqrt{N / n}$-net $\mathcal{N}_{\alpha}$ with respect to the operator norm on $L\left(B_{2}^{n}, \alpha \bar{B}\left(\omega_{1}\right)\right)$ with

$$
\operatorname{card} \mathcal{N}_{\alpha} \leq C_{0}^{n^{2}}
$$

where $C_{1}=C(4)$ is taken from Fact $3.3(\mathrm{i})$ and $C_{0} \geq 0$ is a suitable numerical constant.

REMARK. Observe that the net $\mathcal{N}_{\alpha}$ depends only on $\omega_{1}$ and $\alpha$.

Proof. Fix $\omega_{1} \in \Omega_{1}^{\prime}$ and note that by Fact 2.1,

$$
\left|\alpha \bar{B}\left(\omega_{1}\right)\right| \leq C_{2}^{n}\left|\alpha \mathcal{E}\left(\omega_{1}\right)\right|
$$


where $C_{2}>0$ is a numerical constant. By (4.1) and the Stirling formula we get

$$
\left|\operatorname{conv}\left\{ \pm x_{i} \mid i=1, \ldots, n\right\}\right|=\left|B_{1}^{n}\right| \prod_{i=1}^{n} b_{i, i} \geq c_{0}^{n}\left|B_{1}^{n}\right|,
$$

where $c_{0}>0$ is a suitable numerical constant. By (4.13), for $\omega_{1} \in \Omega_{1}^{\prime}$ we have $\left|\operatorname{det}\left[g_{i}\left(\omega_{1}\right)\right]_{i=1}^{n}\right| \geq(\varrho \sqrt{n / N})^{n}$, where $\varrho=\varrho(4)$ is taken from Fact 3.3. Thus

$$
\begin{aligned}
\left|\operatorname{conv}\left\{ \pm y_{i}\left(\omega_{1}\right) \mid i=1, \ldots, n\right\}\right| & =\left|\operatorname{det}\left[g_{i}\left(\omega_{1}\right)\right]_{i=1}^{n}\right|\left|\operatorname{conv}\left\{ \pm x_{i} \mid 1 \leq i \leq n\right\}\right| \\
& \geq\left(c_{0} \varrho \sqrt{n / N}\right)^{n}\left|B_{1}^{n}\right| .
\end{aligned}
$$

Finally, by (4.14) we have $\left\|y_{i}\left(\omega_{1}\right)\right\|_{2}=\left\|\Gamma_{n, N} x_{i}\right\|_{2} \leq 2 C_{1} \sqrt{n / N}$ for every $i=$ $1, \ldots, n$. Thus Proposition 5.3 in [MT4] and the Remark following it yield the existence of a $\left(1 / 4 C_{1}\right) \sqrt{N / n}$-net $\mathcal{N}_{\alpha}$ in $\mathbf{A}_{\alpha}$ with card $\mathcal{N}_{\alpha} \leq\left(4 C C_{1} C_{2} / c_{0} \varrho\right)^{n^{2}}$, where $C>0$ is a numerical constant.

Proposition 5.2. There exists $\alpha_{0}=\alpha_{0}(\lambda, \kappa, \delta, \varepsilon)$ such that if $\lambda, \kappa, \delta$, $\varepsilon, k, n$ and $l$ are as in Theorem 3.4 and $A\left(\alpha_{0}\right)$ and $\widetilde{B}$ are defined by (4.15) and (4.8) respectively, then for every $\omega_{1} \in \Omega_{1}^{\prime}$ we have

$\mathbb{P}_{2}\left\{\omega_{2} \in \Omega_{2} \mid\right.$ there exists $T \in \operatorname{Mix}_{n}(\kappa n, 1)$ such that

$$
\begin{aligned}
& Q T y_{i}(\omega) \in A\left(\alpha_{0}\right) \widetilde{B}\left(\omega_{1}\right) \text { for every } i=1, \ldots(1+\varepsilon) n \\
& \left.\qquad \text { and } \omega=\left(\omega_{1}, \omega_{2}\right) \in \Omega_{0}\right\} \leq e^{-n^{2}} .
\end{aligned}
$$

Proof. Fix $\omega_{1} \in \Omega_{1}^{\prime}$ and fix $\alpha_{0}>0$ to be specified later. Let $\Theta\left(\alpha_{0}\right)$ be the subset of $\Omega_{2}$ considered in the proposition. For each $T \in \operatorname{Mix}_{n}((\kappa-\varepsilon) n, 1)$ set

$\Theta\left(T, 2 \alpha_{0}\right)=\left\{\omega_{2} \in \Omega_{2} \mid Q T y_{i}\left(\omega_{1}, \omega_{2}\right) \in 2 A\left(\alpha_{0}\right) \widetilde{B}\left(\omega_{1}\right)\right.$ for $\left.1 \leq i \leq(1+\varepsilon) n\right\}$.

We shall split the condition appearing in the definition of $\Theta\left(\alpha_{0}\right)$ into two separate conditions. Since $\omega_{1}$ is fixed so are the $y_{i}$ 's for $1, \ldots, n$, while the $y_{i}$ 's for $i=n+1, \ldots, n+\varepsilon n$ depend on $\omega_{2}$. We shall use the condition

$$
Q T y_{i}\left(\omega_{1}\right) \in A\left(\alpha_{0}\right) \widetilde{B}\left(\omega_{1}\right) \quad \text { for } i=1, \ldots, n
$$

in order to control the cardinality of a net in a suitable set of operators. The second condition

$$
Q T y_{i}\left(\omega_{2}\right) \in A\left(\alpha_{0}\right) \widetilde{B}\left(\omega_{1}\right) \text { for } i=n+1, \ldots, n+\varepsilon n
$$

will provide a probability estimate for individual operators in the net, via Lemma 4.2 .

Let $\mathcal{N}$ be the net in $\mathbf{A}_{A\left(\alpha_{0}\right)}$ constructed in Lemma 5.1.

Claim.

$$
\Theta\left(\alpha_{0}\right)=\bigcap_{S \in \mathcal{N}} \Theta\left(S, 2 \alpha_{0}\right)
$$


Indeed, let $\omega_{2} \in \Theta\left(\alpha_{0}\right)$ and pick $T \in \operatorname{Mix}_{n}(\kappa n, 1)$ such that both (5.1) and (5.2) are satisfied. By a standard "lifting" argument, condition (5.1) implies the existence of an operator $T_{1} \in L\left(\mathbb{R}^{n}\right)$ satisfying

$$
T_{1} y_{i}\left(\omega_{1}\right) \in A\left(\alpha_{0}\right) \bar{B}\left(\omega_{1}\right) \quad \text { for } i=1, \ldots, n, \quad Q T_{1}=Q T .
$$

Hence $T_{1}\left|(\operatorname{ker} Q)^{\perp}=T\right|(\operatorname{ker} Q)^{\perp}$. Since $\operatorname{dim} \operatorname{ker} Q=\varepsilon n$ it is easy to check that $T \in \operatorname{Mix}_{n}(\kappa n, 1)$ implies that $T_{1} \in \operatorname{Mix}_{n}((\kappa-\varepsilon) n, 1)$. Thus $T_{1} \in \mathbf{A}_{A\left(\alpha_{0}\right)}$. Pick $T_{0} \in \mathcal{N}$ with

$$
\left\|T_{1}-T_{0}: B_{2}^{n} \rightarrow A\left(\alpha_{0}\right) \bar{B}\left(\omega_{1}\right)\right\| \leq\left(1 / 4 C_{1}\right) \sqrt{N / n}
$$

and arbitrary $i \in\{n+1, \ldots, n+\varepsilon n\}$. By (4.14), for $\left(\omega_{1}, \omega_{2}\right) \in \Omega_{0}$ we have $\left\|y_{i}\left(\omega_{2}\right)\right\|_{2} \leq 2 C_{1} \sqrt{n / N}$. Hence by $(5.2)-(5.4)$ we get

$$
\begin{aligned}
Q T_{0} y_{i}\left(\omega_{2}\right) & =Q T_{1} y_{i}\left(\omega_{2}\right)+Q\left(T_{0}-T_{1}\right) y_{i}\left(\omega_{2}\right) \\
& \in Q T_{1} y_{i}\left(\omega_{2}\right)+\left(A\left(\alpha_{0}\right) / 2\right) Q\left(\bar{B}\left(\omega_{1}\right)\right) \\
& =Q T y_{i}\left(\omega_{2}\right)+\left(A\left(\alpha_{0}\right) / 2\right) \widetilde{B}\left(\omega_{1}\right) \subset 2 A\left(\alpha_{0}\right) \widetilde{B}\left(\omega_{1}\right),
\end{aligned}
$$

which implies that $Q T_{0} y_{i}\left(\omega_{2}\right) \in 2 A\left(\alpha_{0}\right) \widetilde{B}\left(\omega_{1}\right)$ for every $i \in\{n+1, \ldots$, $n+\varepsilon n\}$. On the other hand, since $T_{0} \in \mathcal{N} \subset \mathbf{A}_{A\left(\alpha_{0}\right)}$ we have $Q T_{0} y_{i}\left(\omega_{2}\right) \in$ $A\left(\alpha_{0}\right) Q(\bar{B})\left(\omega_{1}\right)=A\left(\alpha_{0}\right) \widetilde{B}\left(\omega_{1}\right)$ for every $i=1, \ldots, n$. Hence $\omega_{2} \in \Theta\left(T_{0}, 2 \alpha_{0}\right)$, which concludes the proof of the Claim.

Returning to the proof of Proposition 5.2 note that for each $\omega_{1} \in \Omega_{1}$ the Claim and Lemmas 4.2 and 5.1 yield

$$
\mathbb{P}_{2}\left(\Theta\left(\alpha_{0}\right)\right) \leq \sum_{S \in \mathcal{N}} \mathbb{P}_{2}\left(\Theta\left(S, 2 \alpha_{0}\right)\right) \leq C_{0}^{n^{2}}\left(C_{1}(\lambda, \kappa, \delta, \varepsilon) 2 \alpha_{0}\right)^{\varepsilon l n} .
$$

Since $l \geq \delta n$ we may choose $\alpha_{0}=\alpha_{0}(\lambda, \kappa, \delta, \varepsilon)>0$ small enough to ensure that the right hand side term in (5.5) is less than $e^{-n^{2}}$.

Proof of Theorem 3.4. Since, by (4.4), (4.6), (4.7) and (4.8), we have $\widetilde{B}\left(\omega_{1}\right)=Q\left(\bar{B}\left(\omega_{1}\right)\right)=Q\left(B\left(\omega_{1}\right)+\mathcal{E}\left(\omega_{1}\right)\right)$, where $\mathcal{E}\left(\omega_{1}\right)$ is an $M$-ellipsoid for $B\left(\omega_{1}\right)$ and $B\left(\omega_{1}\right)=Q \Gamma_{n, N,\left(\omega_{1}, \omega_{2}\right)}(K)$, by Corollary 2.2 for every $\omega_{1} \in \Omega_{1}$ we get

$$
\begin{aligned}
W\left(\widetilde{B}\left(\omega_{1}\right),(\kappa-2 \varepsilon) n, l\right) & \geq C_{0}^{-2 / \delta} W\left(B\left(\omega_{1}\right)+\mathcal{E}\left(\omega_{1}\right),(\kappa-2 \varepsilon) n, l\right) \\
& \geq C_{0}^{-3 / \delta} W\left(B\left(\omega_{1}\right),(\kappa-2 \varepsilon) n, l\right) \\
& =C_{0}^{-3 / \delta} W\left(Q \Gamma_{n, N,\left(\omega_{1}, \omega_{2}\right)}(K),(\kappa-2 \varepsilon) n, l\right) \\
& \geq C_{0}^{-5 / \delta} W\left(\Gamma_{n, N,\left(\omega_{1}, \omega_{2}\right)}(K),(\kappa-2 \varepsilon) n, l\right) .
\end{aligned}
$$

Let $\boldsymbol{\Omega}_{1}$ be the subset of $\boldsymbol{\Omega}$ consisting of those $\omega$ for which the inequality in Theorem 3.4 is violated with $c=\alpha_{0} C_{0}^{-5 / \delta}$. Note that if $\omega=\left(\omega_{1}, \omega_{2}\right) \in$ $\Omega_{1} \cap\left(\Omega_{1}^{\prime} \times \Omega_{2}\right)$ then there exists an operator $T \in \operatorname{Mix}_{n}(\kappa n, 1)$ for which 


$$
T y_{i}(\omega) \in c \Gamma_{n, N, \omega}(K) \quad \text { for all } i=1, \ldots, N \text {. }
$$

In particular, applying the projection $Q$ to both sides of (5.7) and using (5.6) we infer that $\omega_{2}$ belongs to the set considered in Proposition 5.2. Thus, by integrating with respect to $\omega_{1} \in \Omega_{1}^{\prime}$ we get

$$
\mathbb{P}\left(\boldsymbol{\Omega}_{1} \cap\left(\Omega_{1}^{\prime} \times \Omega_{2}\right)\right) \leq e^{-n^{2}} .
$$

The proof is concluded by observing that by (4.11) and (4.12),

$$
\mathbb{P}\left(\boldsymbol{\Omega}_{1} \cap\left(\left(\Omega_{1} \backslash \Omega_{1}^{\prime}\right) \times \Omega_{2}\right)\right) \leq 2 e^{-4 n} .
$$

\section{References}

[B] J. Bourgain, On finite-dimensional homogeneous Banach spaces, in: Geometric Aspects of Functional Analysis (1986/87), Lecture Notes in Math. 1317, Springer, $1988,232-238$.

[G1] E. D. Gluskin, The diameter of Minkowski compactum roughly equals n, Funct. Anal. Appl. 15 (1981), 57-58 (English translation).

[G2] - Finite-dimensional analogues of spaces without basis, Dokl. Akad. Nauk SSSR 216 (1981), 1046-1050 (in Russian).

[M1] P. Mankiewicz, Finite-dimensional Banach spaces with symmetry constant of order $\sqrt{n}$, Studia Math. 79 (1984), 193-200.

[M2] - Subspace mixing properties of operators in $\mathbb{R}^{n}$, with applications to Gluskin spaces, ibid. 88 (1988), 51-67.

[MaS] P. Mankiewicz and S. J. Szarek, On the geometry of proportional quotients of $l_{1}^{m}$, ibid. 155 (2003), 51-66.

[MT1] P. Mankiewicz and N. Tomczak-Jaegermann, A solution of the finite-dimensional homogeneous Banach space problem, Israel J. Math. 75 (1991), 129-159.

[MT2] - - - Schauder bases in subspaces of quotients of $l_{2}(X)$, Amer. J. Math. 116 (1994), 1341-1363.

[MT3] -, -, Quotients of finite-dimensional Banach spaces; random phenomena, in: Handbook of the Geometry of Banach Spaces, W. B. Johnson and J. Lindenstrauss (eds.), Vol. II, Elsevier, 2003, 1201-1246.

[MT4] - - - Geometry of families of random projections of symmetric convex bodies, Geom. Funct. Anal. 11 (2001), 1282-1326.

[Mi1] V. D. Milman, Inégalité de Brunn-Minkowski inverse et applications à la théorie locale des espaces normés, C. R. Acad. Sci. Paris Sér. I Math. 302 (1986), 25-28.

[Mi2] —, Surprising geometric phenomena in high-dimensional convexity theory, in: European Congress of Mathematics (Budapest, 1996), Vol. 2, Birkhäuser, 1998, 73-91.

[MiS1] V. D. Milman and G. Schechtmann, Asymptotic Theory of Finite-Dimensional Normed Spaces, Lecture Notes in Math. 1200, Springer, 1986.

[MiS2] -, -, Global versus local asymptotic theories of finite-dimensional normed spaces, Duke Math. J. 90 (1997), 73-93.

[Pi] G. Pisier, Volumes of Convex Bodies and Banach Space Geometry, Cambridge Univ. Press, 1989.

[S1] S. J. Szarek, The finite-dimensional basis problem with an appendix on nets of Grassmann manifolds, Acta Math. 151 (1983), 153-179. 
[S2] S. J. Szarek, On the existence and uniqueness of complex structure and spaces with "few" operators, Trans. Amer. Math. Soc. 293 (1986), 339-353.

[T] N. Tomczak-Jaegermann, Banach-Mazur Distances and Finite-Dimensional Operator Ideals, Pitman Monogr. Surveys Pure Appl. Math. 38, Longman, Harlow, 1989.

Institute of Mathematics

Polish Academy of Sciences

Śniadeckich 8

00-956 Warszawa, Poland

E-mail: piotr@impan.gov.pl
Department of Mathematical and Statistical Sciences

University of Alberta Edmonton, Alberta

Canada T6G 2G1

E-mail: nicole@ellpspace.math.ualberta.ca

Received December 3, 2002

Revised version January 29, 2003 